\title{
Un an après la crise des pénuries de médicaments Sandoz : peu d'avancées
}

Depuis quelques années, on reconnait l'importance du problème engendré par les pénuries de médicaments (aussi appelées ruptures d'approvisionnement de médicaments) au Canada $^{1-14}$. En février 2012, Sandoz Canada annonçait à l'ensemble de sa clientèle canadienne qu'il devait cesser la production de près d'une centaine de produits et qu'il devait interrompre ou réduire provisoirement la production d'environ 200 produits, en réponse à un avis reçu de la Food and Drug Administration ${ }^{15}$. Le Canada n'est pas le seul pays ayant subi une pénurie de médicaments. Une crise similaire, mais touchant davantage certains champs thérapeutiques (p. ex. oncologie) est survenue en Europe ${ }^{16}$ et aux États-Unis au cours des deux dernières années ${ }^{17-22}$.

En 2008, le gouvernement canadien avait été informé du risque de pénurie de médicaments pour la population canadienne et du danger inhérent à la diminution de la concurrence entre les fabricants de médicaments génériques ${ }^{23}$. Avec la crise vécue en 2012, plusieurs intervenants canadiens se sont concertés afin de déterminer les actions susceptibles de prévenir ou de réduire les pénuries de médicaments. Dans le cadre du Séminaire administratif de l'Association des pharmaciens des établissements de santé du Québec tenu en octobre 2012, nous avons fait le point sur les recommandations émises par ces intervenants canadiens au cours de la dernière année. Au 31 mars 2013, nous avons repris cette analyse des actions entreprises afin d'assurer la mise en place de recommandations visant à prévenir les pénuries de médicaments au Canada. Le tableau 1 présente le profil des recommandations par intervenant s'étant exprimé sur le sujet, soit le Comité permanent de la Chambre des communes, le Collège royal des médecins et chirurgiens du Canada, l'Ordre des pharmaciens du Québec, l'Association des pharmaciens du Canada et le groupe d'achat de SigmaSanté. Cette synthèse met en évidence un total de 30 recommandations, que nous avons brièvement reformulées aux fins de notre synthèse, et que nous avons regroupées par thématique, soit gouvernements, groupes d'achats, chaîne d'approvisionnement, industrie pharmaceutique et pharmaciens.

Malgré les nombreuses recommandations proposées par tous ces intervenants, force est de constater, un an après la crise de pénuries de médicaments découlant des activités de Sandoz Canada, qu'un faible nombre d'entre elles ont été mises en 


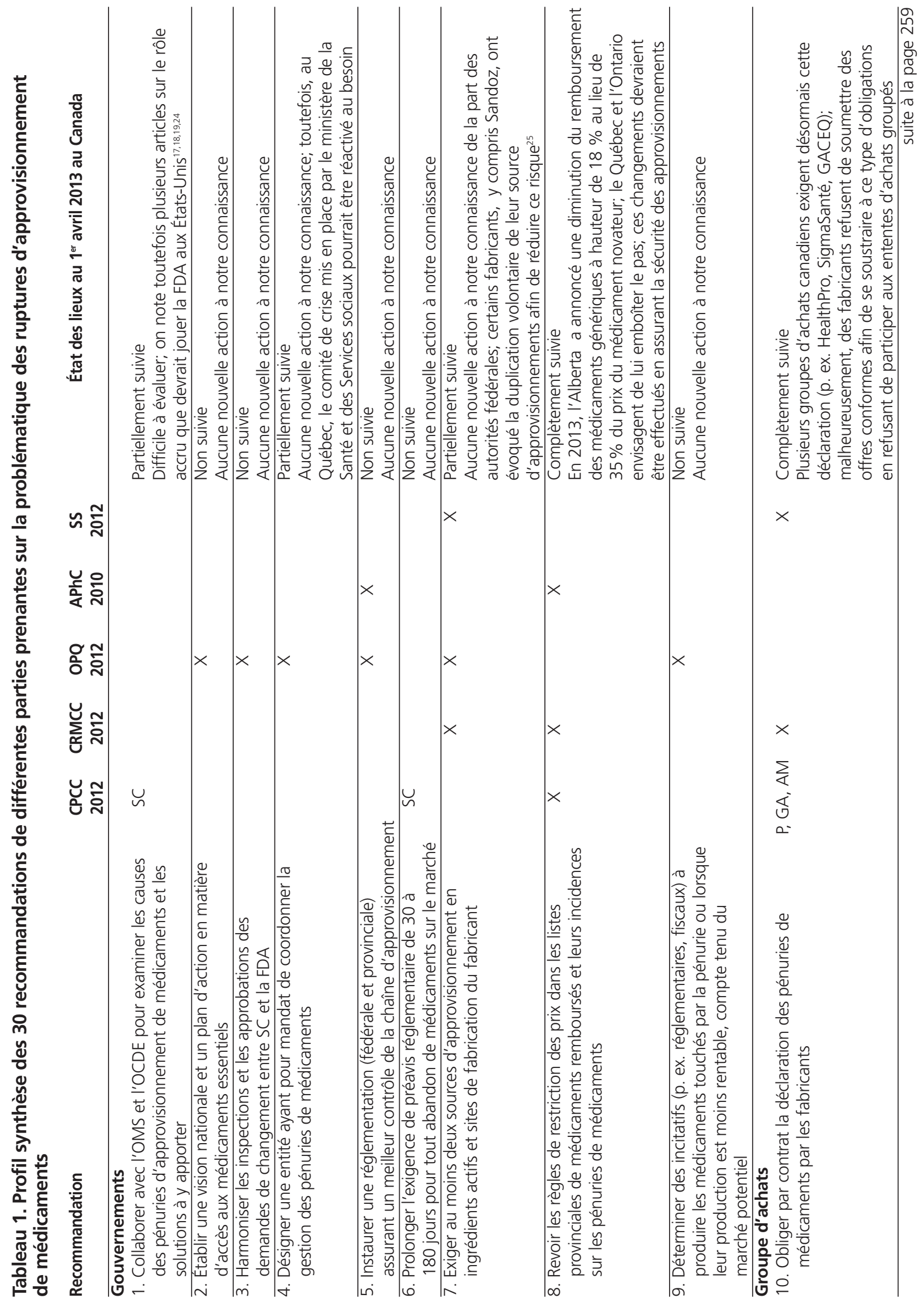




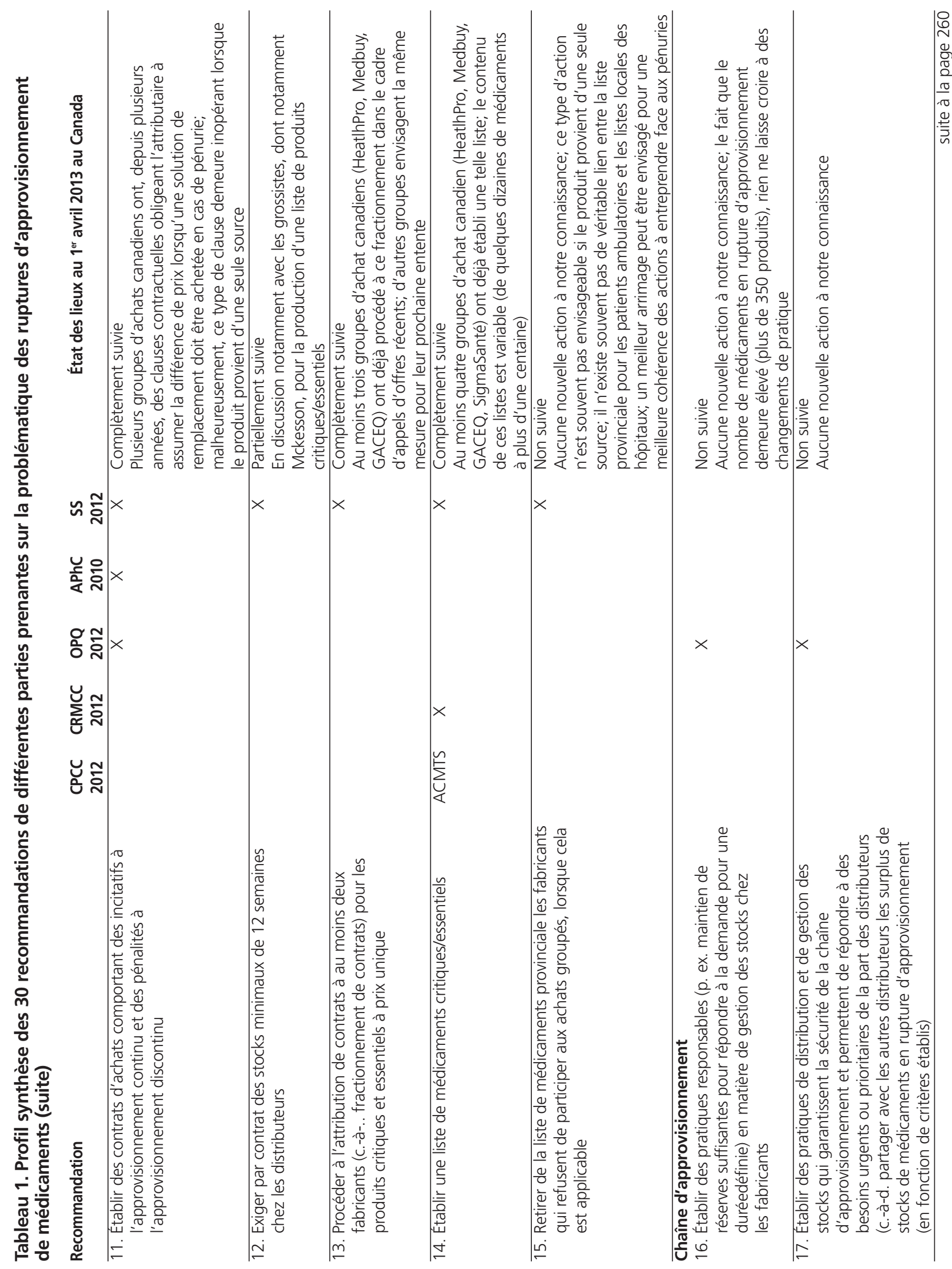




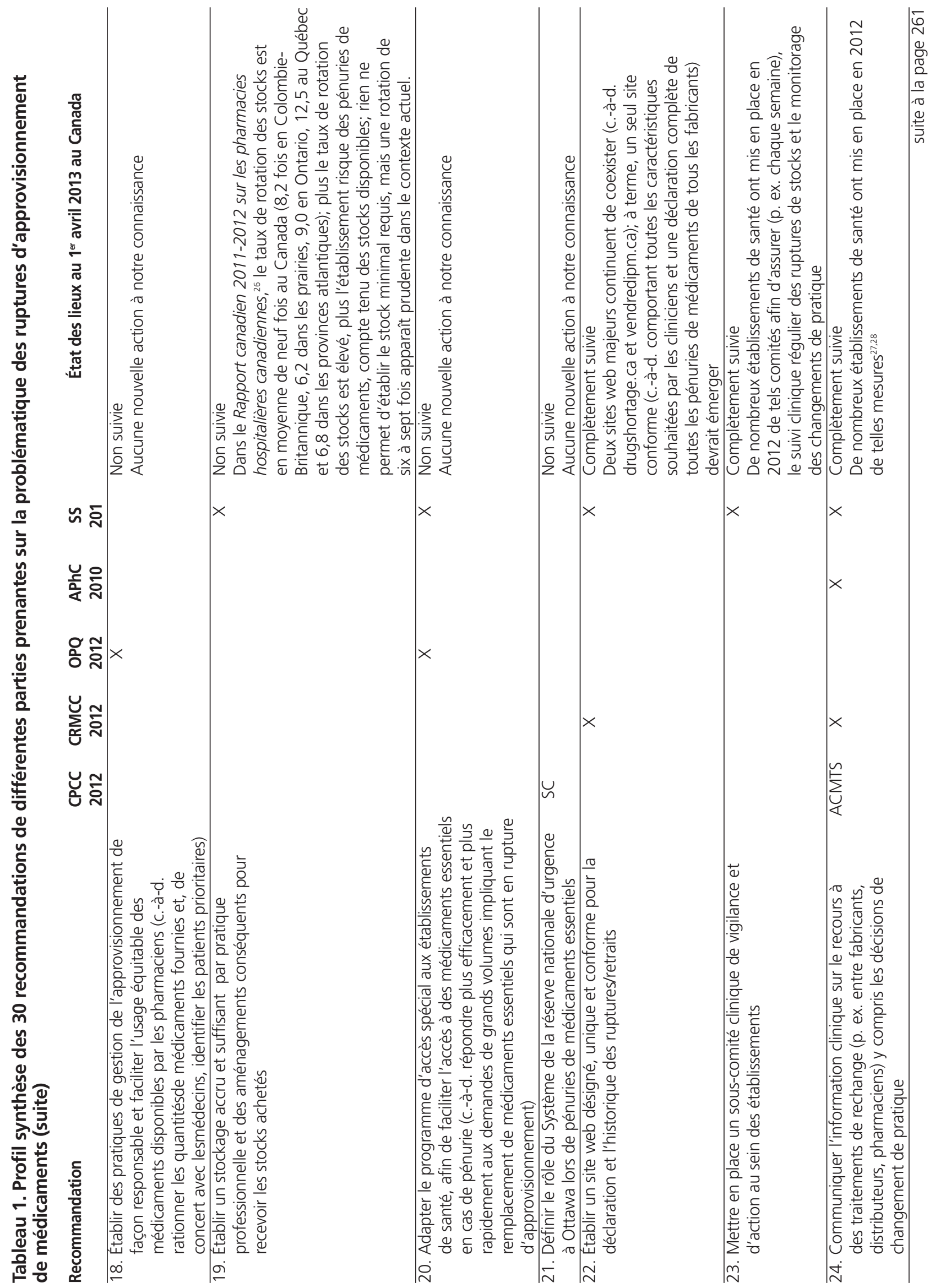




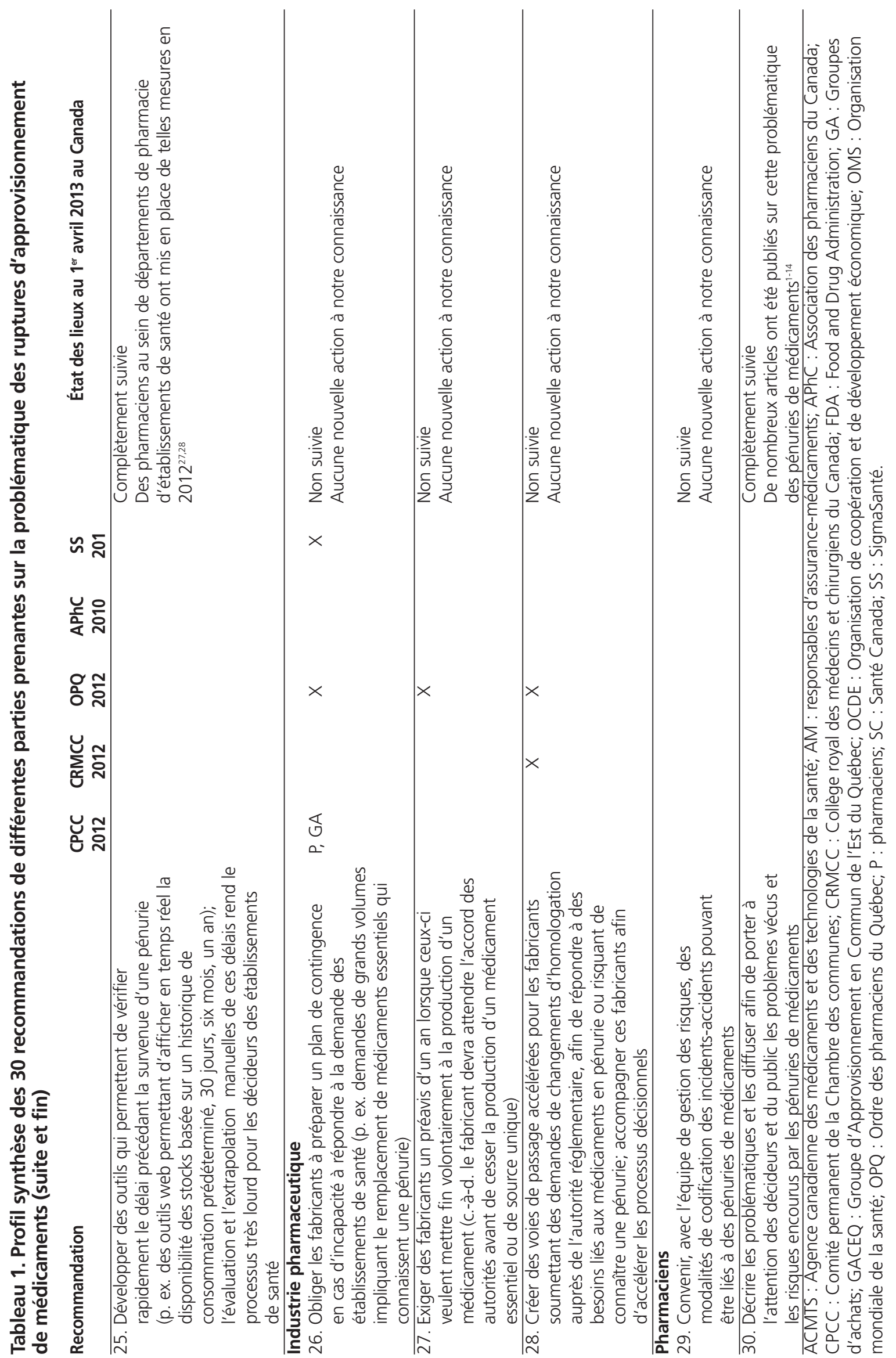


application au Canada. Des 30 recommandations recensées, seulement 10 comportent des actions concrètes identifiées par notre équipe (voir tableau 1 : recommandations 8, 10, 11, 13, $14,22,23,24,25,30)$. On note en outre que certaines recommandations n'ont rencontré qu'un faible écho. Par exemple, en décembre 2012, la ministre fédérale de la Santé a annoncé vouloir conserver le caractère facultatif de la déclaration des pénuries de médicaments, contrairement aux pharmaciens et aux groupes d'achats qui demandaient que la déclaration soit obligatoire $^{29}$. Bien que la gestion électronique des allocations par les grossistes soit reconnue comme une mesure contribuant à réduire les répercussions pour les pharmaciens hospitaliers, seuls les médicaments de Sandoz demeurent gérés en allocation électronique par le grossiste Mckesson ${ }^{30}$. Bien qu'une majorité de fabricants reconnaissent l'importance de la déclaration des pénuries, certains refusent de signer des ententes avec des groupes d'achats afin de se soustraire notamment à l'obligation de déclaration des pénuries de médicaments sur les sites web canadiens destinés à cet effet. Face à ces timides avancées que peut-on conclure? Peut-on parler d'indifférence compte tenu que le plus gros de la crise est passé? Au 31 mars 2013, on comptait plus de 350 médicaments en pénurie sur le site de vendredipm.ca. Une nouvelle lettre d'avertissement de la Food and Drug Administration vient d'être émise à Apotex Canada en février 2013 $3^{31}$. Quelle en sera la portée? Nous pensons que la diffusion de cet état de la situation peut encourager toutes les parties prenantes à continuer les travaux afin d'apporter de réels changements au circuit du médicament en vue de prévenir et de réduire les risques de pénuries de médicaments au Canada.

\section{Références}

1. Bussières JF, Lebel D. Ruptures et retraits de médicaments. Québec Pharm. 2007;54(2):53-4.

2. Bussières JF, Chiveri A, Lebel D. Perspective sur les ruptures d'approvisionnement de médicaments en établissement de santé de 2006 à 2010. Can J Hosp Pharm. 2011;64(6):426-35.

3. Ottino G, Lebel D, Bussières JF, Bourdon O. Gestion des ruptures d'approvisionnement de médicaments :perspectives en France, aux États-Unis et au Canada. Can J Hosp Pharm. 2012;65(1):37-42.

4. Ottino G, Lebel D, Bussières JF. Drug shortages in health care institutions: perspectives in early 2012 [lettre]. Can J Hosp Pharm. 2012;65(2):151-2.

5. Ottino G, Lebel D, Bussières JF. Perspective face aux causes de ruptures d'approvisionnement de médicaments. Pharmactuel. 2012;45(3):207-12.

6. Barthélémy I, Lebel D, Bussières JF. Une solution à vos vendredis après-midi ? vendredipm.ca. Pharmactuel. 2012;45(1):12-3.

7. Barthélémy I, Bussières JF, Lebel D, Gaudreault P, Prot-Labarthe S, Bourdon O. Drug shortages: what does France do better than Canada? [lettre]. Can J Hosp Pharm. 2012;65(5):406-7.

8. Barthélémy I, Lebel D, Bussières JF. Drug shortages in health care institutions: perspectives in early 2013 [lettre]. Can J Hosp Pharm. 2013;66(1):39-40.

9. Barthélémy I, Lebel D, Bussières JF. Quel avenir pour les données portant sur les ruptures d'approvisionnement de médicaments déclarées sur les sites web de surveillance? [lettre]. Can J Hosp Pharm. 2013;66(2):135-6.

10. Barthélémy I, Bussières JF. Impact des ruptures d'approvisionnement de médicaments : perception des pharmaciens d'établissements de santé. Pharmactuel. 2013;46(2)

11. Évaluation des conséquences des ruptures d'approvisionnement en $2012 \mathrm{au}$ sein des cinq $\mathrm{CHU}$ québécois. Programme de gestion thérapeutique des médicaments. Publié au : http://www.pgtm.qc.ca/documentation/FSW/ RUPTURES_AD_20130702.pdf, Consulté le 10 juillet 2013.

12. Barthélémy I, Lebel D, Bussières JF. Ruptures d'approvisionnement de médicaments : profil de l'année 2012 [présentation orale]. Congrès annuel de l'Association des pharmaciens d'établissement de santé du Québec; 24-26 avril 2013; Québec (QC).

13. Barthélémy I, Lebel D, Bussières JF. Les ruptures d'approvisionnement en établissement de santé : un problème de santé publique à l'aube 2013 [affiche].
Colloque annuel de l'association des étudiants et étudiantes en santé publique de l'Université de Montréal; 13 février 2013; Montréal (QC).

14. Barthélémy I, Lebel D, Bussières JF. Perception of the impact of drug shortages on healthcare professionals and patients in Canada. Conférence annuelle sur la pratique professionnelle de la Société canadienne des pharmaciens d'hôpitaux; 3-5 février 2013; Toronto (ON).

15. Lettre aux clients (15-16 février). Boucherville (QC) : Sandoz Canada Inc.; 2012 février 15. Publié au : www.sandoz.ca/site/fr/products/inventaire/ customerletter/letter1.shtml?licensecode=catalogue. Consulté le 6 mai 2013.

16. Huys I, Simoens S. European drug shortages: a call for action! Int J Pharm Pract. 2013;21(1):1-2.

17. Schweitzer SO. How the US Food and Drug Administration can solve the prescription drug shortage problem. Am J Public Health. 2013;103(5) :e10-4.

18. Kuehn BM. Despite curbing new drug shortages, shortfall of drugs a persistent problem. JAMA. 2013;309(6):532-3.

19. Kweder SL, Dill S. Drug shortages: the cycle of quantity and quality. Clin Pharmacol Ther. 2013;93(3):245-51.

20. Gupta DK, Huang SM. Drug shortages in the United States: a critical evaluation of root causes and the need for action. Clin Pharmacol Ther. 2013; 93(2):133-5.

21. Tucker ME. US drug shortages: a disappearing problem? BMJ. 2012; 345:e8551.

22. Moffett BS, Mossad EB. Drug shortages: implications on pediatric anesthesia practice and management resources. J Clin Anesth. 2012;24(8):677-9.

23. Pour une concurrence avantageuse des médicaments génériques au Canada : préparons l'avenir. Ottawa $(\mathrm{ON})$ : Bureau de la concurrence; 2008. Publié au www.bureaudelaconcurrence.gc.ca/eic/site/cb-bc.nsf/vwapj/GenDrugStudyReport-081125-fin-f.pdf/\$FILE/GenDrugStudy-Report-081125-fin-f.pdf. Consulté le 8 avril 2013.

24. Woodcock J, Wosinska M. Economic and technological drivers of generic sterile injectable drug shortages. Clin Pharmacol Ther. 2013;93(2):170-6.

25. Sandoz Canada establishes secure dual manufacturing sites for medically necessary injectable products [annonce médiatique]. Boucherville (QC) : Sandoz Canada; 2012. Publié au www.sandoz.ca/assets/content/en/forms/ pdf/Dual_manufacturing_sites_PR_EN_FINAL_2012-07-24.pdf. Consulté le 8 avril 2013.

26. Doucette D. Systèmes de distribution de médicaments. Dans : Babich M, Bornstein C, Bussières JF, Doucette D, Hall K, Lefebvre P, et al. Rapport annuel 2011-2012 sur les pharmacies hospitalières canadiennes. Eli Lilly; 2012. p. 1827. Publié au www.lillyhospitalsurvey.ca/HPC2/content/2012_report/ chapter_cF\%20.pdf. Consulté le 10 juillet 2013.

27. Barthélémy I, Métras ME, Tataru A, Marchildon-Juneau A et Ouellette Frève Johann-François. Étude de cas de ruptures d'approvisionnement de médicaments en établissements de santé. Risques Qual. [sous presse].

28. Barthélémy I, Lebel D, Atkinson S et Bussières JF. Ruptures d'approvisionnement de médicaments : une illustration de la gestion des risques en établissement de santé. Risques Qual. [sous presse].

29. Volontary drug-shortage reporting adopted despite concerns. Canadian Press; 27 décembre 2012. Publié au www.cbc.ca/news/health/story/2012/12/27/ health-minister-drug-shortage-plan-approval.html. Consulté le 8 avril 2013.

30. Bussières JF, Labelle B et Lussier-Labelle F. Perspectives sur les achats groupés de médicaments en établissement de santé. Ruptures, revue transdisciplinaire en santé. 2005;10(2) :56-72

31. Inspections, compliance, enforcement, and criminal investigations: Apotex warning letter. Silver Spring (MD): Food and Drug Administration; 2013. Publié au www.fda.gov/ICECI/EnforcementActions/WarningLetters/2013/ ucm344476.htm. Consulté le 8 avril 2013.

Isabelle Barthélémy, MD, MHSC, FRCPC, FACP, FACG, FCAHS

Assistante de recherce

Jean-François Bussières, B. Pharm., M. SC., FCSHP

Chef

Unité de recherche en pratique pharmaceutique

Centre hospitalier universitaire Sainte-Justine

Montréal (Québec)

Isabelle Barthélémy est aussi une étudiante (D. Pharm.) à Université Claude Bernard Lyon 1, Villeurbanne, France. Jean-François Bussières est aussi chef du Département de pharmacie au Centre hospitalier universitaire Sainte-Justine et professeur, Faculté de pharmacie, Université de Université de Montréal, Montréal (Québec).

Intérêts concurrents : aucun déclaré. 